\title{
Flexible Bronchoscopy Findings in Children with Congenital Lobar Emphysema: 8-Year Data from a Single Center Study
}

\author{
Gokcen Tugcu ${ }^{1}$, Sanem Eryılmaz ${ }^{1}$, Şule AKYAN SOYDAŞ ${ }^{1}$, Ece Ocak ${ }^{1}$, Murat Gençoğlu ${ }^{1}$, \\ Satı Tabakçı ${ }^{1}$, Salih Uytun ${ }^{1}$, and Guzin Cinel $^{1}$ \\ ${ }^{1}$ Ankara City Hospital
}

April 24, 2021

\begin{abstract}
Introduction:Congenital lobar emphysema (CLE) is a rare developmental lung malformation that involves the hyperaeration of one or more lung lobes due to partial obstruction and occurs at a rate of 1/20,000-30,000 live births. Here,we aimed to retrospectively examine the clinical, radiological, and bronchoscopy findings of patients with CLE who were diagnosed and treated with surgical or conservative approaches in our clinic to compare our results with those in the literature. Method:We examined the clinical, and radiological data and FB findings of the patients with CLE aged 0-18 years at our center between 2013 and 2020.We also examined the symptoms and findings recorded during the patients' follow-up. Results:.The median age of 20 patients with CLE at diagnosis was 3.2 years (range, 1 day-17 years). Respiratory distress and mediastinal shift were more prominent in the patients who underwent surgery than the patients who were followed up conservatively and diagnosed at an early age $(\mathrm{p}=0.001, \mathrm{p}=0.049, \mathrm{p}=0.001$, respectively). Discussion: In line with studies in the literature, the pulmonary symptoms and CLE-related imaging findings in our study regressed during the conservative follow-up.We recommend clinicians consider performing a detailed anamnesis for patients with unresolved respiratory symptoms and unilateral or bilateral increased ventilation,along with appropriate imaging tests and examinations, and should consider CLE in the diagnosis.
\end{abstract}

\section{INTRODUCTION}

Congenital lobar emphysema (CLE) also known as congenital lobar overinflation, is a rare developmental lung malformation. The respiratory system begins to develop during the third week of pregnancy, and developmental anomalies during this stage can lead to parenchymal lung malformations (1-3). CLE is the hyperaeration of one or more lung lobes due to partial obstruction (4) and occurs at a rate of 1/20,000-30,000 live births. CLE is more common in male infants but rarely causes respiratory distress in newborns (5-7). The diagnosis can be performed with ultrasonography at various stages of the pregnancy (8). Approximately half of the patients with CLE are symptomatic at birth, and most are diagnosed within the first 6 months of life (9-11). The most affected lobes are the left upper lobe, right upper lobe, and right middle lobe. Involvement of the lower lobes and more than one lobe is very rare (9-13). Pathology samples typically show no tissue damage, except for acquired emphysema and the occasional accompanying cartilage hypoplasia. Acinar and alveolar structures are both normal in CLE; however, the alveoli can experience secondary hypertrophy, and the affected lung tissue cannot postnatally mature (9). Bronchial collapse develops due to structural damage in the bronchial wall and can cause distal air trapping on expiration (14). The etiology of CLE can include hypoplasia, dysplasia, and the absence of primary or secondary bronchial cartilage; however, most of these conditions are idiopathic. CLE can be observed as the result of congenital bronchial diseases such as primary bronchial stenosis/atresia and bronchomalacia. Secondary causes of CLE include meconium aspiration and the obstruction of mucous plaques and membranes (15-17). Although rare, bronchogenic cysts and mediastinal masses have also been shown to cause CLE (18). The progressive increase in ventilation can also result in compression of adjacent organs and, rarely, impaired perfusion. Substernal and subcostal 
retraction, wheezing, cyanosis, and feeding difficulties can occur during the neonatal period, whereas chronic cough and frequent lung infections can occur during infancy and might be difficult to distinguish from foreign body aspiration (9).

During bronchial development, as cardiac structures develop between 4 and 6 weeks of pregnancy, CLE can also be accompanied by congenital heart diseases (CHDs), such as patent ductus arteriosus, atrial septal defect, ventricular septal defect, total anomalous pulmonary venous drainage, and tetralogy of Fallot at varying rates. External compression of vascular structures can sometimes be the cause of secondary CLE (19). Hyperechogenicity, mediastinal shift, and polyhydramnios can be observed through intrauterine ultrasonography of the lung structures, with normal blood flow (20-23). During the postnatal period, the first choice for diagnosis is chest radiography (CXR), which can reveal increased aeration in the affected lobe. In severe cases, the CLE might be accompanied by herniation of the opposite lung (9). Thoracic computed tomography (CT) performed with contrast provides more detailed information on vascular compression, endobronchial occlusive masses, bronchial atresia, etc., in elucidating the causes and differential diagnosis. Anatomical anomalies such as bronchial atresia, bronchomalacia, mucus plaques, and mucosal flaps can be observed with flexible bronchoscopy (FB). Suspected foreign body aspiration can also be ruled out with FB (23).

Treatment decisions are reached according to clinical, radiological, and bronchoscopy findings. When the symptoms and signs are mild to moderate, when the patient's growth and development are not affected, and when there is no accompanying respiratory distress, a conservative approach is adopted. Lobectomy is recommended only for patients with severe and progressive findings (23). Close follow-up is recommended for patients for whom a conservative approach is advised, given that the clinical findings can progress at later ages and that surgery might be required (5).

The aim of this study is to retrospectively examine the clinical, radiological, and bronchoscopy findings of patients diagnosed with CLE in our clinic who are followed up with conservative and/or surgical approaches and to compare our results with those in the literature.

\section{METHODS}

This research was planned as a retrospective descriptive study. We examined the demographic, clinical, and radiological data and FB findings of the patients with CLE aged 0-18 years diagnosed at the Ankara Child Health and Diseases Hematology Oncology SUAM in Ankara, Turkey, between January 2013 and September 2019 and at Ankara City Hospital between September 2019 and December 2020. We also examined the symptoms and findings recorded during the patients' follow-up.

\section{Statistics and Ethics Approval}

The data obtained during the study are expressed as percentages. We employed the SPSS 21.0 program for the statistical analysis, using the chi-squared test or Fisher's exact test to determine the categorical variables and the non-parametric Mann-Whitney U test to determine the numerical variables. Ethics approval was obtained from our center's Ethics Committee (No E2-21-224).

\section{RESULTS}

Twenty-one patients were diagnosed with CLE at our center between 2013 and 2020. One patient did not attend the follow-up meetings; therefore, 20 patients were ultimately included in the study. The median age at diagnosis was 3.2 years (range, 1 day-17 years), and the median age at admission to our clinic was 4.53 years (range, 21 days-17 years). Nine of the patients (45\%) were female, and 11 (55\%) were male. Seven (35\%) patients had no symptoms at admission to our clinic. Fourteen patients $(87.5 \%)$ presented with cough, and peroral antibiotics were administered with the diagnosis of pneumonia; before admission to our clinic.

Two patients (10\%) were diagnosed with CXR, and thorax ultrasonography was performed during the postnatal period, with cystic lesions observed in the lungs in the fetal ultrasound. Three patients (15\%) were diagnosed with suspected lung infection and moaning during the neonatal period, four patients (20\%) were 
diagnosed with foreign body aspiration, and nine patients (45\%) were diagnosed with recurrent and/or intractable lung infection.

One patient $(5 \%)$ had a normal CXR and was diagnosed after a trauma. One patient was diagnosed with incidental CLE by CT performed because of cavities in the lung after pneumonia. With the exception of these two patients, all patients presented with localized increased aeration on the CXR. In the CXR and CT, two patients (10\%) presented with mediastinal shift and secondary atelectasis, and three patients $(15 \%)$ presented with mild mediastinal/tracheal deviation. All but three patients (15\%) underwent thoracic CT before FB. On CT, left upper lobe 10 (50\%), right middle lobe 3 (15\%), right upper lobe 2 (10\%), left lingula $1(5 \%)$, left lower lobe 1 (5\%) which may be compatible with CLE. There were two patients (\%10) with multiple lesions which may be compatible with CLE. One $1(5 \%)$ patient with right emphysematous areas which were observed in the upper and right middle lobes, and 1(5\%) patient with bilateral emphysematous areas in the left lower and right upper lobes. CT demonstrated vascular compression in one (5\%) patient (Table 1). None of the patients underwent ventilation-perfusion scintigraphy.

All the patients underwent FB to rule out primary and secondary causes that could lead to increased aeration. Macroscopically, FB revealed malacia in eight patients (40\%), mucosal folds in seven patients (35\%), and fish-mouth deformity in five patients $(25 \%)$. There was accompanying bronchial atresia in two patients and a mild vascular pulsatile mass in one patient (Table 1). There was no microbiologically significant growth in the bronchoalveolar lavage samples, and the cytopathological examination results were normal.

Two patients $(10 \%)$ who were followed up with a diagnosis of asthma underwent inhaled steroid therapy, and two patients(10\%) were diagnosed with wheezing. One patient (5\%) was diagnosed with tethered cord syndrome, one patient (5\%) was diagnosed with a laryngeal cleft, and one patient (5\%) was diagnosed with familial dystonia; 3 patients (15\%) presented with CHD. Echocardiography revealed patent ductus arteriosus in one patient $(5 \%)$, secundum atrial septal defect in one patient (5\%), and a small muscular ventricular septal defect in one patient (5\%). The patients diagnosed with CHD did not require medical or surgical treatment, and there was no statistically significant difference between the two groups in terms of comorbid diseases (Table 1).

Lobectomy was performed by the surgical department for four symptomatic patients. The first patient was admitted to the pediatric intensive care unit for respiratory distress and pneumonia at the age of three months and for increased aeration in the upper zone of the left lung, causing right upper atelectasis and a mediastinal shift as detected in the patient's CXRs. The patient was admitted due to their need for invasive positive mechanical ventilation. His left upper lobe was markedly malastic as shown by FB, was diagnosed with CLE and underwent left upper lobectomy. While the second patient was being followed up in the neonatal intensive care unit with $2 \mathrm{~L} / \mathrm{min}$ of oxygen support through a nasal cannula, he was referred to our clinic as an outpatient at the age of five months with an total hyperinflation of the left lung that did not improve and caused mediastinal shifting. The patient whose FB revealed a fish-mouth deformity in the left lower lobe underwent a left lower lobectomy. The third patient was examined at four months of age due to coughing and wheezing; increased ventilation was observed in the patient's left lung, causing mediastinal shifting. The FB revealed that the left upper lobe had a fish-mouth shape and that there was a mucosal flap in the lateral part of the lesion. The fourth patient was examined for a lung infection at the age of 15 days and was followed up on an outpatient basis due to increased ventilation in their left lung, causing a mediastinal shift in the patient's CXRs. The FB revealed a significant fish-mouth deformity in the left upper lobe. Both third and fourth patients with mediastinal shift and frequent lung infections underwent left upper lobectomy within two weeks after the diagnosis.

The pathology evaluation of the lobectomy specimens found enlargement of alveolar structures in four specimens and immature bronchial cartilages in two specimens. Concomitant pulmonary sequestration, congenital airway malformation, and malignancy were not observed.

Although most of the patients who underwent surgery were male, there was no statistically significant difference in sex among the patients who were followed up conservatively. The four patients who underwent 
lobectomy at diagnosis had a median age of 1.9 months (range, 1 day-4 months), and the most common symptoms were respiratory distress, tachypnea, and retraction. The patients who were followed up conservatively had a median age of 3.16 years ( 4 days- 17 years) at diagnosis, and the most common symptoms were cough. Of the four patients who underwent surgery, three patients $(75 \%)$ had left upper lobe involvement, and one patient $(25 \%)$ had left lower lobe involvement. The mediastinal shift was observed in all patients, and secondary atelectasis was observed in two patients in the imaging and in four patients who underwent surgery. Respiratory distress and mediastinal shift were more prominent in the patients who underwent surgery than the patients who were followed up conservatively and diagnosed at an early age $(\mathrm{p}=0.001, \mathrm{p}=$ $0.049, \mathrm{p}=0.001$, respectively). Although the rate of left upper lobe involvement was higher for the patients who underwent surgery than for those treated conservatively, the difference was not statistically significant $(\mathrm{p}=0.067 ;$ Table 1$)$.

Of the patients with CLE who were followed up conservatively after diagnosis, four were able to cooperate with the spirometry in the follow-up, one patient had normal spirometry, one had mild obstruction, and two had mild restriction. The patients who underwent surgical lobectomy were aged between 1 and 4 years at their last visit in 2020; therefore, spirometry could not be performed (Table 2).

The median follow-up period was 23.2 months ( 8 months -7 years). In the check-ups of the four patients who underwent lobectomy, their complaints significantly regressed, their growth and development were normal, and they did not experience pneumonia during their follow-up. There were also no changes except for postoperative changes in their CXRs and increased compensatory aeration on the operated side. Sixteen patients who were conservatively followed up had no complaints in their check-ups and did not have pneumonia or asthma attacks. One patient presented with linear atelectasis, which improved with physiotherapy and postural drainage. None of the patients required oxygen, and none were malnourished. In the last visit, the increase in aeration in the check-up CXRs regressed in all patients and was highly evident in two patients(12.5\%; Table 3). None of the patients who were conservatively followed up or who underwent surgery required a repeat $\mathrm{CT}$ in their follow-up. There were no deaths in the early or late postoperative period in any of the patient groups.

\section{DISCUSSION}

This retrospective cross-sectional study found that patients diagnosed with CLE, who underwent surgical lobectomy, were symptomatic compared with the patients with conservative follow-up and those among the former who were diagnosed in the neonatal and early infancy period. The most common symptoms of the patients who underwent lobectomy at presentation were respiratory distress, tachypnea, and retraction. The most common symptom of the patients who were conservatively followed up was coughing. As with studies in the literature, our study observed that the most commonly involved lobes are the left upper and right middle lobes $(5-8,11)$. The CLE is currently defined as a rare congenital airway malformation characterized by lobar hyperinflation that causes respiratory distress in the neonatal and infancy period. Obstruction in bronchial structures, increased aeration, compression atelectasis in adjacent tissues, and mediastinal shift are observed in symptomatic patients who are diagnosed within the first 6 months, mostly during the first postnatal days (24). Early diagnosis (fetal ultrasound, etc.) is important because tension pneumothorax can occur in these patients when respiratory distress develops with the first postnatal inspiration and when positive pressure ventilation is applied (25).

In our study, only two patients were diagnosed with fetal ultrasonography, whose widespread use has resulted in more frequent diagnoses of congenital pulmonary malformations (CPM). A multicenter retrospective study in 2019 examined data from 506 patients with CPM and observed CLE in 53 patients (10.3\%) (26). The diagnosis of CPM without CLE has been shown to be significantly more frequent with fetal ultrasonography than with CLE, a result that has been attributed to the nonspecific echogenicity and rarer occurrence of CLE in fetal ultrasonography (26-28). Asymptomatic CLE with no mediastinal shift or atelectasis is diagnosed late. When diagnosed, this condition is mostly conservatively treated (29-30). CLE can go unnoticed in follow-up ultrasounds and, as it progresses, can sometimes lead to severe respiratory distress at birth (30). The presence of mediastinal shift and atelectasis in $\mathrm{AR}$ at birth has been correlated to respiratory distress 
and the need for neonatal intensive care during the neonatal period. Ten percent of patients with CLE and severe symptoms undergo surgical lobectomy during the neonatal period, mostly in the first week postpartum $(27,28)$. Given that our study was retrospective, detailed fetal ultrasounds were not available for all of the conservatively followed up patients; however, the patients with an incidental diagnosis who were attended by a perinatologist were followed up.

In our study, CLE occurred within a wide age range, from the neonatal period to 17 years of age. In recent years, CLE has been shown to have a wide range of possible symptoms, from minimal pulmonary symptoms to respiratory failure in the neonatal period $(6,13,26)$. These differences can vary depending on the lobar and segmental location of the CLE $(26,31)$. There are reports in the literature of patients diagnosed with incidental segmental CLE at the age of 15 days who had a chest tube inserted with the pre-diagnosis of pneumothorax during the neonatal period and were subsequently diagnosed with CLE $(8,32)$.

In our study, two patients with CLE were diagnosed incidentally in the imaging performed for other reasons, and four patients were diagnosed in the imaging performed for suspected foreign body aspiration and in the FB imaging. A study reported on a patient who was examined with a pre-diagnosis of CLE at the age of 48 days and was found to have foreign body aspiration; there was also a case report of a patient with suspected foreign body aspiration, who was diagnosed with CLE at the age of 15 years $(15,33)$.

In our study, left upper lobe involvement was more common in the surgical group than in the conservatively treated group; however, the difference was not statistically significant. CLE with left upper lobe involvement is more symptomatic and more frequently requires surgery. There are publications showing that patients with right middle lobe CLE are generally asymptomatic and are conservatively treated; however, this might be due to the higher frequency of left upper lobe CLE (34-36).

In line with studies in the literature, the pulmonary symptoms and CLE-related imaging findings in our study regressed during the conservative follow-up. Many studies have shown no lung infection or malignant transformation during conservative follow-up (13,34-36). In the patients who underwent surgery, alveolar emphysema was the most common finding in the pathology specimens, with half of the specimens presenting cartilage hypoplasia. In the literature, emphysematous changes are the most common pathology findings, and accompanying cartilage hypoplasia has less frequently been reported (36).

In our study, the rate of CHD and other accompanying diseases was $15 \%$, which is consistent with studies showing that the incidence of CHD varies between $6 \%$ and $24 \%$ in patients with CLE $(14,37,38)$.

The limitations of our study were: 1) the results of the fetal ultrasounds could not be obtained for all patients due to the study's retrospective design; 2) the patients were followed up with other diagnoses because our hospital is a reference center; and 3) the delay before the diagnosis was relatively prolonged, especially in the patients who underwent surgery. Due to technical issues (e.g., performing spirometry in patients too young to cooperate), detailed pulmonary function tests such as impulse oscillometry could not be performed on all patients.

In conclusion, we observed that respiratory distress and mediastinal shift were more prominent in patients who underwent surgery with a diagnosis of CLE compared with patients who were conservatively followed up. We also observed that those who underwent surgery were diagnosed at an earlier age. Clinicians should consider performing a detailed anamnesis for patients with unresolved respiratory symptoms and unilateral or bilateral increased ventilation. Clinicians should also perform appropriate imaging tests and examinations (including FB) and should consider CLE in the diagnosis.

\section{REFERENCES}

1. Pariente G, Aviram M, Landau D, Hershkovitz R. Prenatal diagnosis of congenital lobar emphysema: case report and review of the literature. J Ultrasound Med. 2009;28(8):1081-1084.

2. Correia-Pinto J, Gonzaga S, Huang Y, Rottier R. Congenital lung lesions underlying molecular mechanisms. Semin Pediatr Surg. 2010;19(3):171-179. 
3. Andrade CF, Ferreira HP, Fischer GB. Congenital lung malformations. J Bras Pneumol. 2011;37(2):259-271.

4. Olutoye OO, Coleman BG, Hubbard AM, Adzick NS. Prenatal diagnosis and management of congenital lobar emphysema. J Pediatr Surg. 2000;35(5):792-795.

5. Thakral CL, Maji DC, Sajwani MJ. Congenital lobar emphysema: experience with 21 cases. Pediatr Surg Int. 2001;17(2):88-89.

6. Mei-Zahav M, Konen O, Manson DC, Langer J. Is congenital lobar emphysema a surgical disease? J Pediatr Surg. 2006;41 (6):1058-1061.

7. Suryawanshi K, Nikumbh D, Singhavi S, Damle R, Dravid N. Congenital lobar emphysema with pulmonary extramedullary hematopoiesis. Turk Patoloji Derg. 2017;33(1):74-76.

8. Cataneo DC, Rodrigues OR, Hasimoto EN, Schmidt Jr AF, Cataneo AJ. Congenital lobar emphysema: 30-year case series in two university hospitals. J Bras Pneumol. 2013;39(4):418-426.

9. Bush A, Chitty LY, Harcourt J, Hewitt RJ, Nicholson AG. Congenital lung disease. In: Wilmott RW, Deterding R, Bush A, Li A, Ratjen F, Sly P, Zar HJ, editors. Kendig's disorders of the respiratory tract in children. 9th ed. Philadelphia (PA): Elsevier, Inc. 2019;321-322.

10. Kravitz RM. Congenital malformations of the lung. Pediatr Clin North Am. 1994;41(3):453-472.

11. Ankermann T, Oppermann HC, Engler S, Leuschner I, Von Kaisenberg CS. Congenital masses of the lung, cystic adenomatoid malformation versus congenital lobar emphysema: prenatal diagnosis and implications for postnatal treatment. J Ultrasound Med. 2004;23(10):1379-1384.

12. Stocker JT, Drake RM, Madewell JE. Cystic and congenital lung disease in newborn. Perspect Pediatr Pathol. 1978;4:93-154.

13. Ozcelik U, Göçmen A, Kiper N, Doğru D, Dilber E, Eg Y. Congenital lobar emphysema: evaluation and long-term follow up of thirty cases at a single centre. Pediar Pulmonol. 2003;35(5):384-391.

14. Karnak I, Şenocak ME, Ciftci AO, Büyükpamukçu N. Congenital lobar emphysema: diagnostic and therapeutic considerations. J Pediatr Surg. 1999;34(9):1347-1351.

15. Aslan AT, Yalcin E, Ozcelik U, Ciftci AO, Kiper N. Foreign-body aspiration mimicking congenital lobar emphysema in a forty-eight day old girl. Pediatr Pulmonol. 2005;39(2):189-191.

16. Warner JO, Rubin S, Heard BE. Congenital lobar emphysema: a case with bronchial atresia and abnormal bronchial cartilages. Br J Dis Chest. 1982;76(2):177-184.

17. Saim L, Mohamad AS, Ambu VK. Congenital lobar emphysema: a case with bronchial septum. Int J Pediatr Otorhinolaryngol. 1994;28(2-3):241-246.

18. Khemiri M, Ouederni M, Ben Mansour F, Barsaoui S. Bronchogenic cyst: an uncommon cause of congenital lobar emphysema. Respir Med. 2008;102(11):1663-1666.

19. Demir OF, Hangul M, Kose M. Congenital lobar emphysema: diagnosis and treatment options. Int J Chron Obstruct Pulmon Dis. 2019;14:921-928.

20. Oliver ER, DeBari SE, Horii SC, Pogoriler JE, Victoria T, Khalek N, Howell LJ, Adzick NS, Coleman BG. Congenital lobar overinflation: a rare enigmatic lung lesion on prenatal ultrasound and magnetic resonance imaging. J Ultrasound Med. 2019;38(5):1229-1239.

21. Tsai PS, Chen CP, Lin DC, Liu YP. Prenatal diagnosis of congenital lobar fluid overload. Taiwan J Obstet Gynecol. 2017;56(4):425-431.

22. Biyyam DR, Chapman T, Ferguson MR, Deutsch G, Dighe MK. Congenital lung abnormalities: embryological features, prenatal diagnosis, and postnatal radiologic-pathologic correlation. Radiographics. 2010;30(6):1721-1738.

23. Ulku R, Onat S, Ozçelik C. Congenital lobar emphysema: differential diagnosis and therapeutic approach. Pediatr Int. 2008;50(5):658-661.

24. Haller Jr JA, Golladay ES, Pickard LR, Tepas III JJ, Shorter NA, Shermeta DW. Surgical management of lung bud anomalies: lobar emphysema, bronchogenic cyst, cystic adenomatoid malformation, and intralobar pulmonary sequestration. Ann Thorac Surg 1979;28(1):33-43.

25. Glenski JA, Thibeault DW, Hall FK, Hall RT, Germann DR. Selective bronchial intubation in infants with lobar emphysema: indications, complications, and long-term outcome. Am J Perinatol 1986;3(3):199-204. 
26. Paramalingam S, Parkinson E, Sellars M, Diaz-Cano S, Nicolaides KH, Davenport M. Congenital segmental emphysema: an evolving lesion. Eur J Pediatr Surg. 2010;20(2):78-81.

27. Kunisaki SM, Saito JM, Fallat ME, Peter SD, Kim AG, Johnson KN, Mon RA, Adams C, Aladegbami B, Bence C, Burns RC. Current operative management of congenital lobar emphysema in children: a report from the Midwest Pediatric Surgery Consortium. J Pediatr Surg. 2019;54(6):1138-1142.

28. Babu R, Kyle P, Spicer RD. Prenatal sonographic features of congenital lobar emphysema. Fetal Diagn Ther. 2001;16(4):200-202.

29. Quinton AE, Smoleniec JS. Congenital lobar emphysema - the disappearing chest mass: antenatal ultrasound appearance. Ultrasound Obstet Gynecol. 2001;17(2):169-171.

30. Olutoye OO, Coleman BG, Hubbard AM. Prenatal diagnosis and management of congenital lobar emphysema. J Pediatr Surg. 2000;35(5):792-795.

31. Mei-Zahav M, Konen O, Manson D, Langer JC. Is congenital lobar emphysema a surgical disease?. J Pediatr Surg. 2006;41(6):1058-1061.

32. Santra A, Dutta P, Manjhi R, Pothal S. Congenital lobar emphysema presenting at late childhood: a rare case report. Lung India. 2014;31(3):302-304.

33. Gorostidi F, de Buys Roessingh A, Gonzalez M, Simon C, Monnier Y. Congenital lobar emphysema presenting as an airway foreign body. Int J Pediatr Otorhinolaryngol. 2015;79(12):2450-2452.

34. Ng C, Stanwell J, Burge DM, Stanton MP. Conservative management of antenatally diagnosed cystic lung malformations. Arch Dis Child. 2014;99(5):432-437.

35. Criss CN, Musili N, Matusko N, Baker S, Geiger JD, Kunisaki SM. Asymptomatic congenital lung malformations: is nonoperative management a viable alternative?. J Pediatr Surg. 2018;53(6):10921097.

36. Thakral CL, Maji DC, Sajwani MJ. Congenital lobar emphysema: experience with 21 cases. Pediatr Surg Int. 2001;17(2-3):88-91.

37. Mendeloff EN. Sequestrations, congenital cystic adenomatoid malformations, and congenital lobar emphysema. Semin Thorac Cardiovasc Surg. 2004;16(3):209-214.

38. Abdel-Bary M, Abdel-Naser M, Okasha A, Zaki M, Abdel-Baseer K. Clinical and surgical aspects of congenital lobar over-inflation: a single center retrospective study. J Cardiothorac Surg. 2020;15:1-8.

\section{Hosted file}

table 1.Demographic, clinical, radiological and bronchoscopic findings of patients diagnosed with cong available at https://authorea.com/users/369107/articles/519355-flexible-bronchoscopyfindings-in-children-with-congenital-lobar-emphysema-8-year-data-from-a-single-centerstudy 


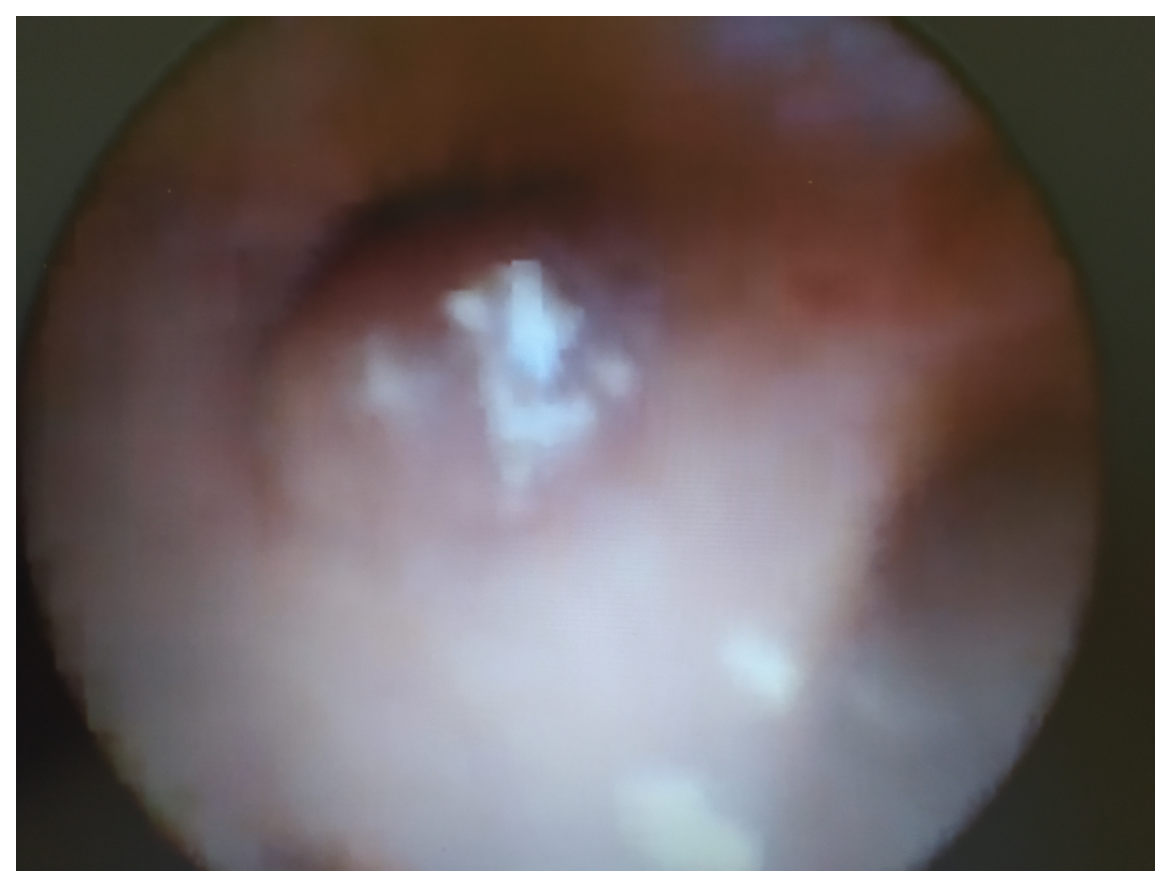

\section{Hosted file}

Table-2_ Follow-up of patients diagnosed with congenital lobary emphysema.pdf available at https://authorea.com/users/369107/articles/519355-flexible-bronchoscopy-findings-inchildren-with-congenital-lobar-emphysema-8-year-data-from-a-single-center-study

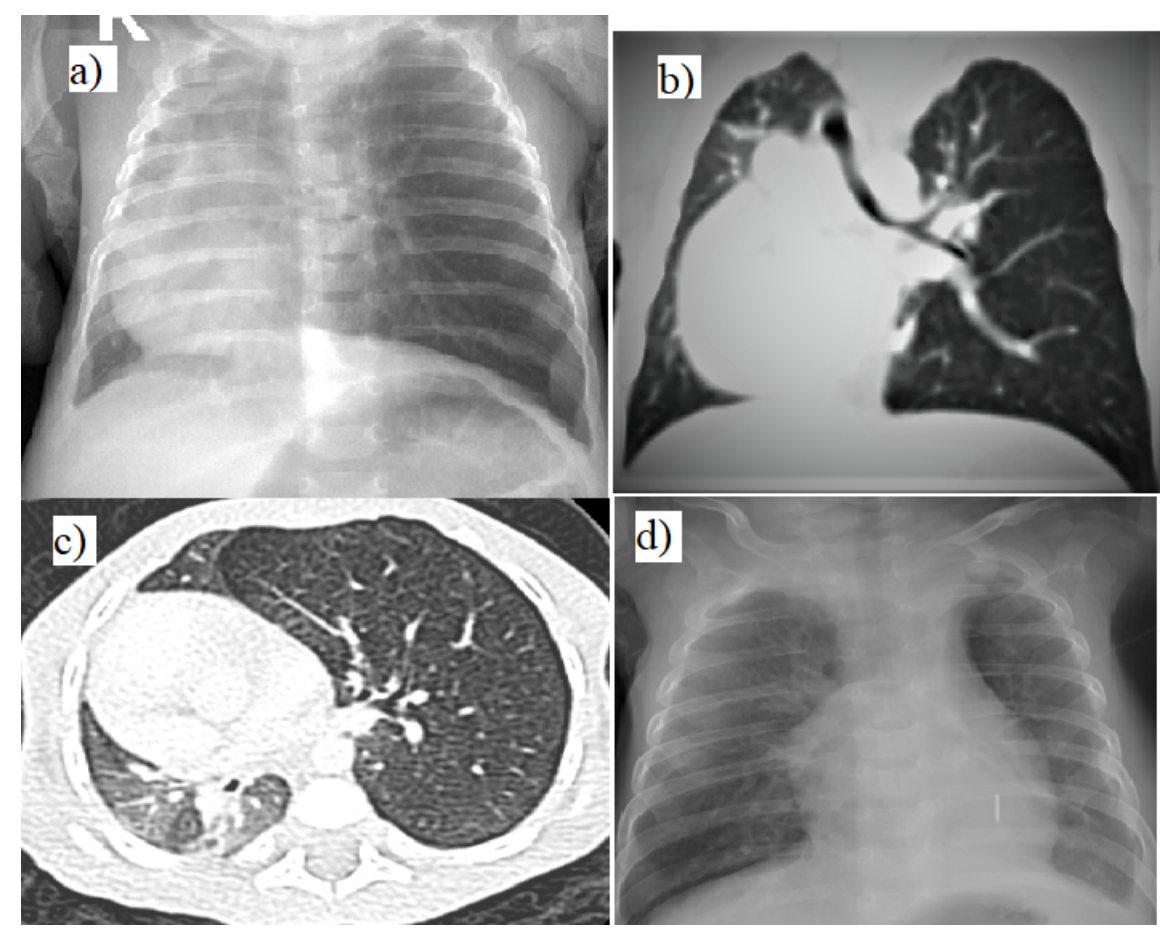




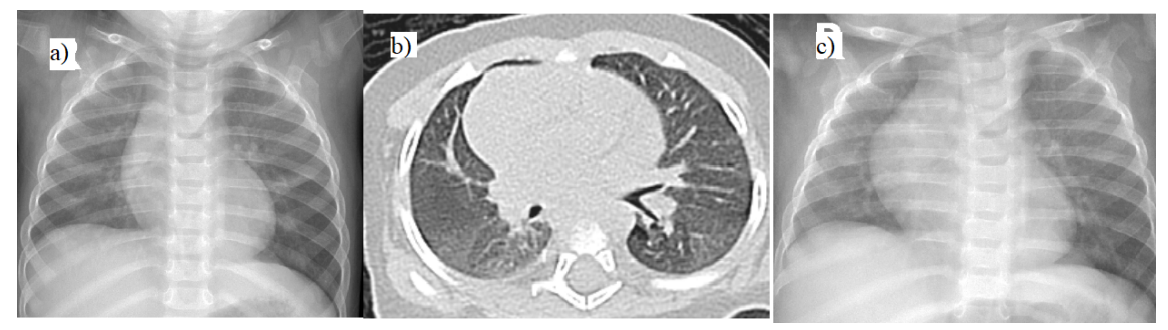

\section{Hosted file}

Table 3.Outcomes of congenital lobar emphysema patients with surgical lobectomy.pdf available at https://authorea.com/users/369107/articles/519355-flexible-bronchoscopy-findingsin-children-with-congenital-lobar-emphysema-8-year-data-from-a-single-center-study 